\title{
Percepção e compreensão dos conceitos contidos na Escala Brasileira de Insegurança Alimentar, em comunidades indígenas no estado do Amazonas, Brasil ${ }^{1}$
}

\section{Perception and comprehension of concepts of the Brazilian Food Insecurity Scale in indigenous communities in the state of Amazonas, Brazil}

\author{
Lucia Kiyoko Ozaki YUYAMA² \\ Victor PY-DANIEL ${ }^{3}$ \\ Noemia Kazue ISHIKAWA 4 \\ Jansen Fernandes MEDEIROS 2 \\ Anne Walleser KEPPLE ${ }^{5}$ \\ Ana Maria SEGALL-CORRÊA ${ }^{5}$
}

\section{R E S U M O}

\section{Objetivo}

Este estudo teve como objetivo avaliar a percepção e a compreensão de conceitos e terminologia da segurança e insegurança alimentar, especialmente os que compõem a Escala Brasileira de Insegurança Alimentar, no contexto da realidade sócio-cultural indígena.

\section{Métodos}

Foram utilizados recursos de pesquisa qualitativa para a abordagem das comunidades indígenas Cacau, Flexeira e Mamori, situadas na bacia hidrográfica do M édio Juruá, nos municípios de Envira e Eirunepé (AM), baseando-se em metodologia já previamente utilizada no Brasil e adaptada ao presente contexto, em uma

\footnotetext{
${ }^{1} 0$ artigo é oriundo do projeto de pesquisa: "Segurança e Insegurança alimentar em Manaus: I. Avaliação epidemiológica; II. Validação de instrumento de coleta de informação em al gumas comunidades indígenas. III. Uso da floresta na promoção de hábitos al imentares saudáveis. Apoio financeiro: Ministério da Ciência e Tecnologia, Ministério do Desenvolvimento Social e Combate à Fome e Conselho Nacional de Desenvolvimento Científico e Tecnológico, a partir do Edital MCT/MESA/ CNPq/CT-Agronegócio 01/2003 (processo CNPq ํ 503431/2003-6).

2 Instituto Nacional de Pesquisas da Amazônia, Coordenação de Pesquisas em Ciências da Saúde. Av. André Araújo, 2936, 69060-001, Manaus, AM, Brasil. Correspondência para/Correspondence to: L.K.O. YUYAMA. E-mail: <yuyama@inpa.gov.br>.

3 Instituto Nacional de Pesquisas da Amazônia, Núcleo de Pesquisa em Ciências Humanas e Sociais. Manaus, AM, Brasil.

4 Instituto Nacional de Pesquisas da Amazônia, Coordenação de Pesquisas em Tecnologia de Alimentos. Manaus, AM, Brasil.

5 Universidade Estadual de Campinas, Faculdade deCiências M édicas, Departamento de M edicina Preventiva e Social. Campinas, SP, Brasil.
} 
reunião com especialistas da área. Em seguida foram organizados grupos focais, com 18 participantes das três comunidades indígenas.

\section{Resultados}

A fome apareceu como situação vivenciada por muitos dos participantes dos grupos focais das três comunidades estudadas. Os conceitos e as terminologias como segurança alimentar, fome e comida boa foram bem compreendidos, no entanto, comida variada, comida suficiente e estratégia para evitar problemas com comida foram conceitos não compreendidos por eles. A rotina de vida desses povos baseia-se nas relações familiares que permitem trocas, diferindo de outros grupos focais da área urbana e rural, conduzidos como parte da validação da Escala Brasileira de Insegurança Alimentar, nos quais a dificuldade de acesso aos alimentos era conseqüência da falta de recursos financeiros.

\section{Conclusão}

São necessários novos e aprofundados estudos, qualitativos e quantitativos, para o desenvolvimento de um instrumento de mensuração de insegurança alimentar que reflita a realidade desses povos, ao mesmo tempo em que busquem fornecer resultados comparáveis com aqueles de outros povos indígenas e mesmo os obtidos pela Escala Brasileira de Insegurança Alimentar em outras populações. Será necessário um instrumento que contemple a questão da troca, o aspecto coletivo, a importância e o uso do ambiente nas experiências de segurança ou insegurança alimentar. Sugere-se ainda que, para estudos quantitativos, o questionário deva ser mais resumido e simples.

Termos de indexação: Insegurança alimentar. População indígena. Segurança alimentar e nutricional.

\section{A B S T R A C T}

\section{Objective}

The objective was to evaluate the perception and comprehension of concepts and terminology related to food security and insecurity, especially those that comprise the Brazilian Food Insecurity Scale, in the context of indigenous socio-cultural reality.

\section{Methods}

Qualitative research techniques were used in Cacau, Flexeira and Mamori indigenous communities located in the M édio Juruá watershed, in the municipalities of Envira and Eirunepé (AM). The methods were based on a methodology used previously in Brazil and adapted to the present context in a meeting of specialists familiar with these indigenous communities. Next, focus groups were organized in each one of the three communities, with a total of 18 participants.

\section{Results}

Hunger appeared as a phenomenon experienced frequently by the participants. Many of the concepts and terms, such as "food security", "hunger", and "good food", were well-understood, but others, such as "varied food", "sufficient food" "strategies to avoid problems with food" were not. Everyday life depends on family relations that allow exchanges, which differs from studies conducted previously in urban and rural areas, where difficulties related to access to food were due to lack of financial resources. The subject of trading, or exchanges, appeared often during the focus groups, as well as a collective aspect of the experience of food security that had not emerged previously in focus groups conducted as part of the Brazilian Food Insecurity Scale validation.

\section{Conclusion}

More in-depth qualitative and quantitative studies are needed to develop a food security measurement instrument that reflects the reality of these indigenous communities while, at the same time, provides results that are comparable to other indigenous communities as well as to results obtained using the Brazilian Food Insecurity Scale in other populations. To apprehend the situation of food security in these grups is necessary an instrument that takes into consideration the question of trading/exchange, the collective aspect, and the importance of the environment in their experiences related to food security and insecurity. It is recommended that, if questionnaires are to be used, that they should be kept as short and simple as possible.

Indexing terms: Food insecurity. Indigenous population. Food security. 


\section{N T R O D U Ç Ã O}

A segurança alimentar se define como 0 direito de todos ao acesso regular e permanente a alimentos de qualidade, em quantidade suficiente, sem comprometer o acesso a outras necessidades essenciais. As políticas e ações de garantia desse direito deverão respeitar as dimensões ambientais, culturais, regionais e sociais ${ }^{1}$. Sendo o direito à alimentação parte dos direitos civis básicos da população e um meio de alcançar a cidadania plena, de acordo com o Instituto Cidadania², impõe-se a necessidade de ações que permitam um recurso adequado para acompanhamento e avaliação das políticas nacionais de combate à fome.

Entre 2003 e 2004, em virtude do compromisso do Estado Brasileiro de priorizar políticas de combate à fome no País, foi realizado estudo multicêntrico para adaptação e validação de escala de medida de insegurança alimentar, com participação de pesquisadores de cinco instituições, Universidade Estadual de Campinas (Unicamp), Instituto Nacional de Pesquisas da Amazônia (INPA), Universidade Federal da Paraíba (UFPB), Universidade Federal de Mato Grosso (UFMT) e Universidade Brasília (UnB). Buscava-se, naquela ocasião, responder à necessidade de identificar, no País, as populações de risco para insegurança alimentar e fome, além de fornecer instrumentos nacionais para o acompanhamento e a avaliação do impacto das políticas públicas pertinentes ${ }^{3,4}$.

A adaptação e a validação desse instrumento partiram de escala desenvolvida pelo United States Department of Agriculture (USDA), e aplicada em inquéritos populacionais naquele país desde 1995, além de em outros países desenvolvidos e subdesenvolvidos ${ }^{6-10}$. No últimos anos, escalas psicométricas da percepção de insegurança alimentar nos domicílios têm alcançado reconhecimento como ferramentas importantes para a avaliação da insegurança alimentar, na sua dimensão de acesso aos alimentos, aliadas aos méto dos mais tradicionais baseados na análise de renda ou de consumo alimentar ${ }^{11,12}$.
A validação da escala no Brasil foi realizada em comunidades urbanas de Campinas, João Pessoa, Brasília e Manaus ${ }^{3}$ e em populações rurais ${ }^{13}$ dos estados correspondentes, acrescidos de $M$ ato Grosso, buscando, dessa forma, captar as diversidades sociais e culturais do Brasil. Entretanto, mesmo tendo em conta as modificações e os esforços na adequação dos questionários para a população de áreas rurais, foi considerado ainda necessário reproduzir o mesmo processo em comunidades indígenas, cujas particularidades e diversidades são bem conhecidas.

Algumas questões nortearam o estudo. Qual a compreensão sobre segurança alimentar para os povos das Terras Indígenas Kulina do Médio Juruá, Kulina do Cacau do Tarauacá, Kanamari do Médio Juruá e Mawetek, e para os pesquisadores familiarizados com essas populações? Pensa-se que seja, sobretudo, a garantia da terra que lhes provê os alimentos por meio da caça, pesca e roçados ${ }^{14,15}$.

Este estudo visa a contribuir com novos conhecimentos a respeito da percepção e da compreensão de conceitos relativos à segurança alimentar contidos na Escala Brasileira de Insegurança Alimentar (EBIA), em comunidades indígenas, por meio de grupos focais conduzidos com membros dessas comunidades. Este esforço poderá resultar, juntamente com outros estudos, na elaboração de um instrumento de medida de fácil aplicação e útil para o aprimoramento das políticas públicas de combate à fome nas populações indígenas, procurando sempre contemplar as especificidades de cada etnia.

\section{M É TO D O S}

0 presente estudo teve aprovação do Comitê de Ética do Instituto Nacional de Pesquisas da Amazônia (Registro 019/2004).

0 procedimento metodológico utilizado para avaliar a compreensão dos conceitos e das terminologias contidos no instrumento de medição da segurança e insegurança alimentar, nas comunidades indígenas, foi viabilizado a partir de 
recursos de pesquisa qualitativa, tendo como referência experiências prévias dos trabalhos de Corrêa et al. ${ }^{13}$, e iniciado com um painel de especialistas que antecedeu os grupos focais nas aldeias.

\section{Painel de especialistas}

O painel de especialistas foi composto por pesquisadores e docentes que atuam em terras indígenas envolvendo as etnias Terena, Universidade Federal do Mato Grosso do Sul (UFMS), Comunidades Yanomami, Kulina e Kanamari e outras etnias, INPA, assim como a Unicamp. Foram discutidos e adaptados conceitos contidos em questionário utilizado por Corrêa et al. ${ }^{13} \mathrm{em}$ áreas urbanas e rurais, com o objetivo de analisar sua pertinência para o diagnóstico da situação de insegurança alimentar em populações indígenas.

Os especialistas sugeriram, com base nas suas convivências com os povos indígenas, algumas modificações nos termos usados no questionário de referência, como as que seguem: alimentação saudável (por comida boa); alimento suficiente (por comida suficiente); qualidade dos alimentos (por comida de qualidade); alimentação variada (por comida variada); fome e segurança alimentar. Durante a discussão foram inseridos outros conceitos tais como alguns dos trabalhados para a área rural: condição para ter alimento suficiente (por condição para ter comida suficiente); troca de alimento (por troca de comida); produção de alimentos para consumo/autoconsumo; compra de alimentos (por compra de comida); ficar sem nenhum dinheiro; estratégias rurais para evitar problemas com alimentação (para estratégias para evitar problemas com comida); poucos alimentos; dinheiro suficiente; alimento nutritivo; comida forte; comida fraca.

Uma vez que, nos hábitos de algumas comunidades indígenas, os adultos se alimentam primeiro, assim como a alimentação é diferenciada de acordo com as atividades de cada membro, foi incluída a pergunta: na hora de servir o prato, quem come primeiro?

\section{Grupos focais}

Com o intuito de viabilizar um estudo exploratório com algumas comunidades indígenas, dois grupos étnicos, os Kulina (autodenominados M adijá), lingüisticamente pertencentes à família Arawá, e os Kanamari (autodenominados Tûkûna), lingüisticamente classificados na família Katukina, foram convidados a participar do presente estudo. No Brasil os Kulina são encontrados em rios da bacia hidrográfica do Purus, em rios da Bacia hidrográfica do Juruá e na Terra Indígena do Javari; enquanto os Kanamari são encontrados no Juruá, no Japurá, no Jutaí e na Terra Indígena do Javari. Segundo a Operação Amazônia Nativa $(\mathrm{OPAN})^{16}$, a população total dessas duas áreas somava 1661 pessoas. Algumas comunidades de ambas as etnias que estão mais próximas dos centros urbanos apresentam mudanças na sua estrutura sociocultural, sobretudo no que se refere aos seus padrões alimentares. Atualmente preferem fazer suas compras nas cidades, incorporando produtos industrializados como açúcar, sal, café, macarrão, bolachas e arroz e até o consumo de bebidas alcoólicas, em vez de basicamente utilizarem produtos de seus roçados ${ }^{14,15}$.

Os grupos focais foram organizados em três comunidades localizadas nas terras indígenas da Bacia Hidrográfica do M édio Juruá, no Sudoeste do estado do Amazonas. Foram elas as comunidades: 1) Cacau (etnia Kulina), localizada na Terra Indígena Cacau do Tarauacá, no M unicípio de Envira, distando $1200 \mathrm{~km}$ em linha reta de M anaus; 2) Flexeira e 3) Mamori (ambos da etnia Kanamari), localizadas na Terra Indígena Kanamari do M édio Juruá, no M unicípio de Eirunepé, distando 1 160km em linha reta de Manaus.

Os conteúdos propostos nos painéis dos especialistas foram explorados nas dinâmicas dos grupos focais. Participaram dessas atividades sete membros da comunidade Cacau, sendo quatro mulheres e três homens; cinco membros em Flexeira, três mulheres e dois homens e seis membros em Mamori, dos quais cinco eram mulheres e um homem. Os participantes dos grupos focais eram todos voluntários indicados pelas lideranças 
da comunidade: Cacique/Capitão, professores ou agentes de saúde.

Os conceitos e os temas eram expostos pelo entrevistador em voz alta, de maneira pausada, sendo sua forma de apresentação repetida ou modificada, quando isso era necessário.

\section{RESULTA D OS E DISCUSSÃ O}

As comunidades estão localizadas em áreas de difícil acesso, necessitando transporte aéreo entre M anaus e as sedes dos M unicípios e, posteriormente, deslocamento das sedes às comunidades, utilizando tratores ou o transporte fluvial denominado "voadeiras" (embarcações pequenas de alumínio motorizadas), seguido por caminhadas de várias horas pela floresta.

Os dois grupos étnicos (Kulina e Kanamari), anteriormente seminômades, lingüisticamente preservados, ainda conservam sua cultura, no entanto suas moradias já estão caracterizadas como a dos ribeirinhos (casas palafitas, predominantemente de palmeiras paxiúba (Socratea exorhiza (mart.) H. Wendland). Seus hábitos alimentares ainda têm como base principal a caça e o cultivo de roçados familiares ou comunitários.

A Comunidade Cacau (na Terra Indígena Kulina do Cacau do Tarauacá), localizada a uma distância reduzida da cidade de Envira, é uma das que apresentam maior deterioração ambiental, tanto em seu espaço como no da vizinhança. Uma das conseqüências é o uso de produtos alimentares estragados, como fonte de alimentação, obtidos em um lixão a céu aberto ao longo da estrada percorrida pelos indígenas.

A Comunidade Mamori (na Terra Indígena Kanamari do Médio Juruá), por sua proximidade à cidade de Eirunepé, apresenta um fluxo intercultural permanente, além de possuir em seu núcleo habitacional uma $M$ issão Evangélica das Novas Tribos do Brasil.

A Comunidade Flecheira (na Terra Indígena Kanamari do Médio Juruá), entre as três estudadas, é a que apresenta certo isolamento em relação às cidades; no entanto, confronta permanentemente a ocupação da margem esquerda do igarapé Itucumã, com assentamentos não indígenas.

Essas comunidades recebem o apoio direto da Organização Não-Governamental (ONG) Operação Amazônia Nativa (OPAN), com sede em Cuiabá e do Núcleo da OPAN em Eirunepé (AM).

\section{Percepções sobre insegurança alimentar}

A insegurança alimentar é uma realidade diária para milhões de pessoas em todo o mundo, e tem constituído tema de pesquisa a busca de um instrumento de medida direta dessa condição que assegure comparações entre países, ou entre comunidades com contextos, costumes, valores e conhecimentos extremamente variados culturalmente. Pesquisas e revisões recentes apontam para essa possibilidade a partir da identificação de aspectos da experiência da insegurança alimentar e da fome considerados universais ${ }^{17}$.

Do mesmo modo que em outras publicações ${ }^{4,13}$, a insegurança alimentar e a fome apareceram como situação vivenciada, freqüentemente, por muitos dos participantes dos grupos focais: "Dói a barriga" , "Quando não tem comida para passar a fome", "Quando não tem o que comer eu choro", "Não ter rancho e morre de fome", "Quando não tem dinheiro, passa assim mesmo, sem comer".

A rotina de vida dos indígenas das comunidades estudadas independe de valores econômicos, por mais que eles tenham de se adequar aos limites financeiros. Estes indígenas estão inseridos em um contexto sócio-histórico comum e, em alguma medida, sem excluir os conflitos, partilham de valores, linguagem e práticas sociais que permitem trocas. A economia interna da aldeia e o suprimento de suas necessidades mais fundamentais são baseadas em relações familiares que permitem trocas, diferindo, portanto, das populações urbanas ${ }^{3}$ e rurais $^{13}$, nas quais a dificuldade de acesso aos alimentos era conseqüência da falta de recursos financeiros. A questão da troca apare- 
ceu com freqüência nas falas durante os grupos focais: "Entregar comida para a vizinha parenta e ganha dela também", "Troca macaxeira com bolacha" , "O marido traz carne de caça, vai para a cidade e troca por peixe", "Um dia dá o peixe e no outro dia ganha carne", "Se a gente tem um tipo de comida, dá para a outra pessoa, aí ela dá outra comida diferente", "Troca peixe por anzol, sal, açúcar, linha para pescar".

Foi possível perceber, também, um aspecto coletivo importante da experiência de segurança alimentar que não surgiu em outros grupos focais conduzidos com populações não indígenas, como parte da validação da EBIA 3,13. "Comida de qualidade é quando a gente rala banana grande verde, mistura com carne cozida e chama todos da comunidade para comer", "[Pouca comida é quando] não dá para os outros, só dá para a família" , "[Pouca comida é] quando não dá para dividir", "A roça da comunidade é uma só".

Esse aspecto coletivo da segurança alimentar merece atenção especial na elaboração de instrumento para medir segurança alimentar dos povos indígenas brasileiros.

Segurança alimentar para as comunidades indígenas está mais centrada nas possibilidades diárias, e não nas possíveis perspectivas a longo prazo, pois os itens alimentares, nas comunidades, são fornecidos por meio de sazonalidade (disponibilidade de frutos oriundos da floresta ou os cultivados em quintais) e encontros casuais (em relação às fontes protéicas, à caça e à pesca). Segurança alimentar para as etnias Kulina e Kanamari é, sobretudo, garantia da terra (para caça, pesca e roçados) como se percebe nas seguintes falas:

- "Quando tem muita pupunha para comer e para vender",

- "Não se preocupar com comida nas épocas de safra porque podem comer e vender",

- "Roçado bom e grande com muita planta é segurança alimentar",

- "Segurança também é material para fazer o roçado como: machado, terçado, enxada, plantadeira de arroz e milho, faca...",
"Pesca no igarapé",

“Pesca de canoa: pacú, surubim, curumatã, piau",

"Planta mamão, abacaxi, pupunha, biriba... macaxeira, banana",

"Cria galinha, porco e boi" ,

"Tem que ter roça grande com milho, mamão, abacaxi, batata doce, caju, macaxeira, banana, cará, manga, ária (um tipo de batata), taioba".

Portanto, é notória a diferença encontrada em relação à compreensão desses participantes, sobre os conceitos relacionados à (in) segurança alimentar contidos na EBIA, e daqueles dos municípios dos estados de São Paulo, Goiás, Paraíba, Amazonas e Mato Grosso, envolvendo populações urbanas e rurais ${ }^{13}$. Essas diferenças estão muito presentes nas questões relativas ao acesso aos alimentos. Nas referidas áreas urbanas e rurais, os resultados revelam que a dificuldade de acesso aos alimentos eram decorrentes da falta de recursos financeiros ${ }^{3,13}$. Além disso, o conceito "segurança alimentar" para os participantes desses grupos focais estava diretamente relacionado com o "acesso às condições de trabalho, saúde, educação, habitação e renda", bem como com "Direito à alimentação". Também estabeleceram associações em relação à " quantidade adequada" da alimentação, mencionando a importância dos aspectos da "qualidade" ; ou seja, "condições de acesso diário e com qualidade" e a "produção de alimentos com qualidade", livres de agrotóxicos e higienicamente seguros, na área rural ${ }^{4,5}$.

Como existem diferenças nos aspectos dos cuidados com as crianças entre as etnias Kulina e Kanamari, não podem ser generalizadas as interpretações relacionadas com segurança alimentar e insegurança alimentar a elas referidas, apesar de se observar que a necessidade de disponibilidade alimentar para as crianças é percebida como semelhante nesses grupos.

O Quadro 1 apresenta uma síntese descritiva dos resultados dos grupos focais, mostrando semelhanças e diferenças de compreensão entre os temas propostos e entre as diferentes etnias. 
Quadro 1.Percepção e compreensão de conceitos e terminologia da segurança e insegurança alimentar em três comunidades indígenas no Amazonas, 2005.

\begin{tabular}{|c|c|}
\hline \multirow{2}{*}{ Conceito } & Comunid \\
\hline & Cacau - Envira - Etnia Kulina \\
\hline 1. Segurança alimentar & $\begin{array}{l}\text { Todo tempo com bucho cheio. } \\
\text { - Quando tem muita pupunha para co- } \\
\text { mer e para vender. } \\
\text { - Na época de açaí, buriti, patauá.. } \\
\text { - Não se preocupar com comida nas } \\
\text { épocas de safra porque podem comer } \\
\text { e vender. }\end{array}$ \\
\hline $\begin{array}{l}\text { 2. Qualidade do ali- } \\
\text { mento (qualidade } \\
\text { da comida) }\end{array}$ & $\begin{array}{l}\text { "A comida que não comemos", "a co- } \\
\text { mida dos brancos","Carne com maca- } \\
\text { xeira", "Peixe assado com caiçuma", } \\
\text { "Carne assada", "Arroz com carne". }\end{array}$ \\
\hline $\begin{array}{l}\text { 3. Alimento saudável } \\
\text { (comida boa) }\end{array}$ & $\begin{array}{l}\text { "Carne de boi com macarrão"; " Arroz, } \\
\text { galinha...";" Caiçuma"; " farinha", "car- } \\
\text { ne cozida...", "Farinha é comida boa", } \\
\text { "Sal não presta". }\end{array}$ \\
\hline $\begin{array}{l}\text { 4. Alimentação variada } \\
\text { (comida variada) }\end{array}$ & $\begin{array}{l}\text { Não compreenderam a palavra "varia- } \\
\text { da" - a pergunta foi reformulada para } \\
\text { "bastante tipo de comida" " Conserva } \\
\text { com farinha de carne.", "M ilho não pres- } \\
\text { ta com sal só com açúcar.", " Fubá com } \\
\text { açúcar", "Não comem verduras.", "M e- } \\
\text { lancia" " batata", "jerimum", "Feijão } \\
\text { preto só presta com sal". }\end{array}$ \\
\hline
\end{tabular}

5. Alimento suficiente (comida suficiente)

6. Comida forte

\section{Comida fraca}

8. Pouca comida

9. Condição para ter Alimento Suficiente (condição para ter comida suficiente)

10.Troca de alimentos (troca de comida)
"Enche a barriga e o resto guarda.", "Sobra comida", "Quando consegue guardar".

"Comida que come e agüenta trabaIhar", "Carne de caça", "Comida que o marido come e fica forte", "cará", "banana grande madura".

Não foi perguntado nesta comunidade.

"Que não dá para os outros, só dá para a família", "Quando não dá para dividir".

"Pesca no igarapé", "Pesca de canoa: pacú, surubim, curumatã, piau.", "Planta mamão, abacaxi, pupunha, biriba", "Planta macaxeira, banana" "Cria galinha, porco e boi".

"Entregar comida para a vizinha parenta e ganhar dela também", "Troca macaxeira com bolacha", "O marido traz carne de caça, vai para a cidade e troca por peixe".
"O que comem todo dia é comida "Mingau de banana boa", "A comida nova que acabou com caldo de peixe", de fazer, porque esta não dói a barri- "Banana grande", ga", "Abacaxi", "pupunha", "aba- "Comida gostosa, a cate", "A comunidade acha que as mais melhor", "Mingau frutas nossas são boas.", "Açaí é de banana." " "Comida comida boa depois de comer carne e com vitamina". pirão com caldo de carne".

Não entenderam a palavra variada. Não entenderam a palaQuando foi explicado como "bastante vra variada.A pergunta tipo de comida", eles falaram que já foi reformulada para tinha sido falado e se irritaram com a "Quando vocês sentem repetitividade das perguntas. que tem bastante tipo de comida?" "Quando tem carne, macaxeira, abacaxi, batata doce, cará, mamão, abacate, ingá...".

Não compreenderam a palavra "sufi- Não compreenderam a ciente". palavra "suficiente"

"Carne de veado", "Carne de anta", "Mingau de banana", "Macaxeira", "Macaxeira com car- "Caiçuma", "O mingau ne", "Carne de porco do mato", de banana é forte por"Carne de queixada". que sua depois", "Pupunha com carne", "Garapa de cana de açúcar".

"Comida não temperada", "Sem "Macaxeira com peixe", sal", "Farinha não tem vitamina", "É "Comida que come só o que não tem vitamina". para assentar a barriga".

"Quando tem pouca comida a gente "Quando tem pouca emagrece.", "Porque a gente está gente tem pouca comimagra.", "Quando passa horas sem da", "Macaxeira com comer".

peixe"

"Faz roça", "Se não plantar nada pas- "Tem que ter roça gransa fome". de com milho, mamão, abacaxi, batata doce, caju, macaxeira, banana, cará, manga, ária (um tipo de batata), taioba"

"Um dia dá o peixe e no outro dia "Troca peixe poranzol, sal, ganha carne", "Se a gente tem um açúcar, linha para pescar.", tipo de comida, dá para a outra pes- "Um traz macaxeira e trosoa, aí ela dá outra comida diferente". ca com banana". 
Quadro 1.Percepção e compreensão de conceitos e terminologia da segurança e insegurança alimentar em três comunidades indígenas no Amazonas, 2005.

\begin{tabular}{ll}
\hline Conceito & Cacau - Envira - Etnia Kulina \\
\cline { 2 - 3 } & \\
\hline 11. Produção de Ali- & Não compreendem a palavra "consu- \\
mentos para consu- & mo". A pergunta foi reformulada para \\
mo (auto-consumo) & "De onde vem o alimento que chega \\
& em sua casa?" "A comida vem do mato", \\
& "Da roça", "A comida vem do merca- \\
& do", "O peixe vem da água".
\end{tabular}

12. Compra de alimentos (compra de comida)

13. Ficar sem nenhum dinheiro

14. Estratégias rurais para evitar problemas com alimentação (Estratégias para evitar problemas com comida).

15. Fome

16. Na hora de servir o prato, quem come primeiro
"Quando não tem dinheiro, passa assim mesmo, sem comer", "Vai matar caça", "Vai pescar, come e o resto vende na cidade", "Aí tem que trabalhar", "Vai pega buriti, açaí, bacaba no mato".

Não compreendem as palavras "estratégias" e "evitar", a pergunta foi reformulada para "O que vocês fazem para não faltar comida?" "Planta macaxeira, banana e pupunha para não passar fome". (Alguns se irritaram com a repetitividade da pergunta e não responderam).

\section{di-}

\section{de}

\section{ar-} "Compra anzol e linha de plástico para pescar"," Compra panela para cozinhar", "Compra panela grande para fazer caiçuma".

"Se ficar sem dinheiro trabalha de novo", "Aí passa fome", "Se precisa de dinheiro faz mais vassoura e artesanato", "Se tiver dinheiro não faz mais, se não tiver faz bolsa, anel de caroço que traz do mato, rede de tucum (este dá mais dinheiro)".

Não compreendem as palavras "estratégias" e "evitar", a pergunta foi reformulada para "O que vocês fazem para não ter problemas com comida?" "Cria galinha e pato", "Não criam porcos, porque dá doença, o porco defeca no terreiro, a criança senta e pega doença.", "Quando chove a água carrega o coco para a beira do rio", "Guarda a semente bem seco para o bicho não mexer no inverno e quando chega o verão planta".

"Dói a barriga", "Quando não tem comida para passar a fome", "Quando não tem o que comer eu choro", "Não ter rancho e morre de fome".

"Quando o marido chega com comida, eu como primeiro porque já estou chorando de fome, depois o marido, depois as crianças" " Todos comem juntos." "O caçador come primeiro, depois junta todas as pessoas e comem", "Os adultos e crianças comem todos juntos, dividindo tudo".

"É ter vontade de comer", "Quando não faz comida fica com fome", "A gente vê pessoa com fome aí dividi comida".

"Pajé e rezador comem separados primeiro", "O restante come todos juntos", "Se tem pouco, todos comem pouco, se tem bastante todos comem bastante". conclusão Mamori - Eirunepé - Etnia Kanamari

Não compreendem a palavra "consumo". A pergunta foi reformulada para "O que é produzido na roça?" - Tudo que foi falado no item 7. (Alguns se irritaram com a repetitividade da pergunta e não responderam.).

"Só compra farinha de macaxeira e milho quando falta aqui".

(1)

"Se não tem dinheiro faz troca".

Não compreendem as palavras "estratégias" e "evitar", a pergunta foi reformulada para "O que vocês fazem para não ter problemas com comida?" "Lava bem o peixe para não pegar doença, "Esquentar bem a comida", "Não comer comida velha", "Se não fizer estas coisas dá dor de barriga diarréia e fica magro".

"Quando a mulher não foi no roçado e não tem o que comer", "Não ter nem banana nem macaxeira", "Quando o homem não vai pescar".
"Todos comem juntos". 
Alguns conceitos sugeridos, pelo grupo de especialistas, sobre 0 instrumento de coleta de dados, foram adaptados no momento da discussão com os participantes dos grupos focais, tais como: comida variada por bastante tipo de comida, ou ainda trabalhar o conceito vários tipos de comida por comidas diferentes; alimento nutritivo por comida que deixa forte e comida que deixa fraco; e consumo/autoconsumo por de onde vem 0 alimento que chega a sua casa e o que é produzido na roça.

Como não houve compreensão do termo estratégia para evitar problemas com alimentação, foi necessário trabalhar no grupo com a pergunta: o que vocês fazem para não faltar e para não ter problemas com comida? Constatase que a percepção do grupo focal em relação à frase faltar comida está mais relacionada à segurança alimentar por meio do uso da terra, envolvendo o plantio e a criação de animais de pequeno porte, de acordo com as falas: planta macaxeira, banana e pupunha para não passar fome; cria galinha e pato, enquanto que o ter problemas com comida está associado ao conceito de alimento seguro (higiene, qualidade), conforme as falas:

- “Não criam porcos, porque dá doença, 0 porco defeca no terreiro, a criança senta e pega doença.",

- "Lava bem o peixe para não pegar doença",

- "Esquentar bem a comida",

- "Não comer comida velha",

- "Se não fizer estas coisas dá dor de barriga diarréia e fica magro".

Os participantes não compreenderam as palavras: suficiente e evitar. Suas percepções em relação aos conceitos trabalhados é que eles são muito repetitivos, deixando-os, às vezes, muito irritados. Todas essas observações apontam para a importância de procurar trabalhar esses conceitos de outra forma, talvez sem se limitar a frases preestabelecidas, para deixar os termos e significados surgirem das falas, de forma mais natural.
Outro aspecto observado foi de que 0 homem e a mulher das etnias Kulina e Kanamari possuem visões distintas em função das atividades e responsabilidades exercidas por eles.

Esforços do governo nas áreas indígenas estão focados, principalmente, na demarcação de terras e ações de saúde, estas últimas contemplando o processo de implantação de Distritos Sanitários. Entretanto, os diversos projetos de saúde implantados não chegam a formular consenso mínimo de como os serviços de saúde seriam melhor organizados ${ }^{18,19}$. Reconhece-se que tanto a problemática da demarcação das terras quanto a da saúde indígena estão fortemente relacionadas com os hábitos e as necessidades alimentares, tornando a disponibilidade de uma metodologia para coleta de informações sobre segurança e insegurança alimentar de fundamental importância.

Este trabalho mostrou, ainda, a necessidade de reformular o questionário de maneira a obter um formato mais resumido e simples, permitindo a sua adaptação às rotinas de trabalho de campo. Isso porque não se pode deixar de considerar a dificuldade de acesso às comunidades, os altos custos de recursos humanos e logísticos de trabalho nessas áreas, os altos índices de analfabetismo nas comunidades e, sobretudo, as dificuldades de compreensão da língua falada.

Athias $\&$ Machado ${ }^{18}$ relatam a necessidade, por ser facilitador, do trabalho em conjunto entre os agentes de saúde e os antropólogos, neste caso específico, em relação às ações de saúde indígena. 0 presente estudo fortalece a visão da existência de carência de espaços de discussões entre profissionais de diferentes áreas, especialmente para a abordagem dos temas sobre a segurança e insegurança alimentar envolvendo as diferentes etnias, de forma a contribuir nas ações das políticas públicas do governo.

\section{O N C L U SÃ O}

Os resultados deste estudo demonstraram que há necessidade de ser desenvolvida meto- 
62 L.K.O. YUYAMA et al.

dologia de abordagem e um instrumento de coleta de informação sobre segurança e insegurança alimentar adequados à realidade social, ambiental, cultural e de gênero, das populações indígenas brasileiras. As adequações no formato de perguntas e na linguagem dos conteúdos do questionário original, validado para populações urbanas e rurais, feitas a partir das análises no grupo de especialistas sobre o instrumento de coleta de dados, e depois nas discussões dos grupos focais, mostraram-se pertinentes, porém ainda insuficientes. É necessário re-estudar o conhecimento dessas comunidades sobre segurança e insegurança alimentar, principalmente com as suas interações ambientais, pois isso fornecerá o substitutivo do aspecto financeiro. Ou seja, deve-se buscar um instrumento de medida adequado às etnias Kulina e Kanamari, explorando a importância e o uso do ambiente nas suas experiências de segurança ou insegurança alimentar e contemplando alterações nos itens que poderiam refletir esse aspecto.

Estudos futuros devem procurar elaborar formas alternativas de abordar os temas de interesse da segurança alimentar, sem limitar a discussão estritamente aos conceitos e termos preestabelecidos, como são aqueles contidos na EBIA. Dessa forma, poderão emergir das falas dos participantes indígenas outros conceitos, referências e significados que melhor refletirão a realidade dos povos indígenas.

Conclui-se que este foi um passo importante de pesquisa sobre segurança e insegurança alimentar entre povos indígenas, que mostrou a existência de várias dimensões da experiência de insegurança alimentar entre eles, já contidas na EBIA. Entretanto, reconhece-se que são necessários novos e aprofundados estudos, qualitativose quantitativos, para o desenvolvimento de um questionário adequado, curto, de linguagem simples. Seus conteúdos devem refletir a realidade dos Kulina e Kanamari, ao mesmo tempo em que contemplem a possibilidade de fornecer resultados comparáveis com aqueles de outros povos indígenas, e mesmo com os obtidos pela EBIA em outras populações.
COLABORADORES

L.K.O. YUYAMA participou do estudo multicêntrico idealizado por Ana M aria Segall-Corrêa et al. e coordenou o projeto aprovado pelo Conselho Nacional de Desenvolvimento Científico e Tecnológico que redundou na redação deste trabalho. V. PY-DANIEL responsável e um dos executores dos levantamentos de campo nas áreas indígenas; contribuiu com a interpretação da percepção indígena em relação à insegurança alimentar. N.K. ISHIKAWA participou da elaboração e aplicação dos questionários nas áreas indígenas; colaborou na análise dos dados e na redação do artigo. J.F. MEDEIROS contribuiu com as atividades de campo nas áreas indígenas e com a interpretação dos dados. A.W. KEPPLE contribuiu com a interpretação dos dados qualitativos e com a redação do artigo. A.M. SEGALL-CORRÊA participou da discussão do conteúdo da investigação que deu origem ao artigo. Contribuiu com a revisão de conteúdo e com a redação do artigo.

\section{RE FE RÊ N C I A S}

1. Brasil. Conferência Nacional de Segurança Alimentar e Nutricional. Documento de referência da II Conferência Nacional de Segurança A limentar e Nutricional. Brasília: CONSEA; 2004.

2. Brasil. Instituto Cidadania. Projeto Fome Zero: uma política de segurança alimentar para o Brasil. São Paulo; 2001.

3. Corrêa AMS, Escamilla RP, Maranha LK, Sampaio MFA. Relatório Técnico. Acompanhamento e avaliação da segurança alimentar de famílias brasileiras: validação de metodologia e de instrumento de coleta de informação (Urbano). Campinas: Unicamp; 2003.

4. Sampaio MFA, Kepple AW, Corrêa AMS, Oliveira JTA, Panigassi G, Maranha LK, et al. Segurança alimentar: experiência de grupos focais com populações rurais do Estado de São Paulo. Segurança Alim Nutr. 2006; 13(1):64-77.

5. United States Department of Agriculture. Food security in the United States: history of the food security measurement project [cited 2003 Aug 22]. Available from: <http:www.ers.usda.gov/briefing/ foodsecurity/history/>.

6. Pérez-Escamilla R. Experiência internacional com a escala de percepção de insegurança alimentar. Cad Est Desenv Soc Debate. 2005; (2):14-27.

7. Melgar-Quinonez HR, Zubieta AC, Valdez E, Whitelaw B, Kaiser L. Validation of an instrument 
to monitor food insecurity in Sierra de Manantlán. Salud Pública Méx, Jalisco. 2005; 47(6):413-22.

8. Melgar-Quinonez HR, Zubieta AC, MkNelly B, Nteziyaremye A, Gerardo MRD, Dunford C. Household food insecurity and food expenditure in Bolívia, Burkina Faso, and the Phillippines. J Nutr [Serial on the Internet]. 2006; 136(Suppl):1431-7 [cited 2006 Oct 21]. Available from: <http:// jn.nutrition.org $>$.

9. Coates J, Frongillo EA, Rogers BL, Webb P, W ilde $P E$, Houser R. Commonalities in the experience of household food insecurity across cultures: What are measures missing? J Nutr [Serial on the Internet]. 2006; 136(Suppl):1438-48 [cited 2006 Oct 21]. Available from: <http://jn.nutrition.org>.

10. Radimer KL. Measurement of household food security in the USA and other industrialised countries. Public Health Nutr. 2002; 5(6A):859-64.

11. Food and Agriculture Organization of the United Nations. Measurement and assessment of food deprivation and undernutrition. International Scientific Symposium, Rome; 26-28 June, 2002. Available from: <http://www. fivims. net/EN/ISS. htm>.

12. Webb P, Coates], Frongillo EA, Rogers BL, Swindale A, Bilinsky, P. Measuring household food insecurity: why it's so important and yet so difficult to do. J Nutr [Serial on the Internet] 2006; 136(Suppl):1404-8 [cited 2006 Oct 21]. Available from: <http://jn.nutrition.org>.

13. Corrêa AMS, Escamilla RP, Maranha LK, Sampaio MFA. Relatório técnico. Acompanhamento e avaliação da segurança alimentar de famílias brasileiras: validação de metodologia e de instrumento de coleta de informação (Rural) (Versão preliminar). Campinas: Unicamp; 2004.

14. Lima D, Py-Daniel V. Áreas indígenas Kanamari do M édio Juruá e Katukina do rio Biá. Levantamento etnoecológico. Brasília: PPTAL/FUNAI; 2002.

15. M onteiro RM, Freitas RA, Py-Daniel V, Gonçalves LG. Diagnóstico etnoecológico e econômico (T.I. Kulina do Médio Juruá, Cacau do Tarauacá (Kulina) e Mawetek (Kanamari). Programa de Fomento a Iniciativas Sustentáveis no Médio Juruá. OPAN (Cuiabá-MT). 2005.

16. Operação Amazônia Nativa. Caderno de atividades - Relatórios. Cuiabá; 2002.

17. Coates J, Frongillo EA, Rogers BL, Webb P, Wilde $P E$, Houser R. Commonalities in the experience of household food insecurity across cultures: what are measures missing? J Nutr. 2006; 136 (Suppl): 1438S-48S.

18. Athias R, Machado M. A saúde indígena no processo de implantação dos Distritos Sanitários: temas críticos e propostas para um diálogo interdisciplinar. Cad Saúde Pública. 2001; 17(2):425-31.

19. Diehl EE. Agravos na saúde Kaigang (Terra Indígena Xapecó, Santa Catarina) e a estrutura dos serviços de atenção biomédica. Cad Saúde Pública. 2001; $17(2): 439-45$

Recebido em: 24/4/2007

Versão final reapresentada em: 3/11/2007 Aprovado em: 8/5/2008 
